\title{
A novel physiologically-inspired method for myoelectric prosthesis control using pattern classification
}

\author{
Strahinja Dosen, Gauravkumar K. Patel, Claudio Castellini, Janne M. Hahne, and Dario Farina
}

\begin{abstract}
The contemporary myoelectric prostheses are advanced mechatronic systems, but human-machine interfacing for robust control of these devices is still an open challenge. We present a novel method for the recognition of user intention based on pattern classification which is inspired by the natural coordination of multiple muscles during hand and wrist motions. The coordinated activation produces a characteristic distribution of the amplitude features of the electromyography signals, and the novel method establishes the class boundaries to capture this natural distribution. The method has been tested in healthy subjects operating a prosthesis during a challenging functional task (bottle grasping, turning and releasing). The novel approach outperformed the commonly used benchmark (linear discriminant analysis), while using shorter training and fewer features. Further developments can, therefore, lead to a method that is suitable for practical implementation and allows robust and efficient control.
\end{abstract}

\section{INTRODUCTION}

$\mathrm{H}$ UMAN hands are indispensable for dexterous execution of daily life tasks. They are also sophisticated instruments for haptic exploration of the environment as well as communication through gestures. Therefore, when the hands are amputated, the person losses many important motor functions. The missing functions can be somewhat restored by using a myoelectric prosthesis. The contemporary prostheses are advanced robotic systems with multiple controllable degrees of freedom (DoFs).

To control these systems, the electrical signals from the user muscles (EMG) are translated into prosthesis commands [1]. The simplest approach is to directly map the signals from an antagonistic muscle pair to the velocity of the motor movement in two directions of a single DoF. To change between the DoFs, the user needs to produce a switching signal. This is a robust but also slow and nonintuitive approach, especially when used to operate a multifunctional prosthesis. Pattern classification can be used to

The authors would like to acknowledge the financial support by the German Ministry for Education and Research (BMBF) under the project INOPRO (16SV7657) and by the EU project INPUT (H2020-ICT-2015687795).

S. Dosen is with the Faculty of Medicine, Department of Health Science and Technology Center for Sensory-Motor Interaction, Fredrik Bajers Vej 7, 9220 Aalborg, Denmark (email: sdosen@hst.aau.dk)

G. Patel and J. Hahne are with the Applied Surgical and Rehabilitation Technology Lab, Department of Trauma Surgery, Orthopedics and Plastic Surgery, University Medical Center Göttingen, 37075 Göttingen, Germany.

C. Castellini is with the Institute of Robotics and Mechatronics, DLR German Aerospace Center, D-82234 Wessling, Germany.

D. Farina is with the Department of Bioengineering, Imperial College London, London, UK avoid the need for the switching and sequential control. This approach exploits the fact that each movement (e.g., grasp type) has a specific myoelectric signature, which can be recorded using multichannel EMG. A classifier is then trained to map the recorded EMG to the corresponding movement intention of the user. This is a potentially effective approach to prosthesis control, and the first commercial system is now available; however, there are still challenges to its wider use due to a lack of robustness during clinical applications [2].

Normally, the only information that is used to develop a myoelectric controller using pattern classification is the input and output data. Although the mapping between the recorded EMG (input) and the motor intention (output) is defined by the underlying physiology of muscle activation, this information is not considered explicitly by the classifier. In the present study, we propose a novel method for pattern classification which directly embeds the properties of muscle physiology and neural control. The hypothesis is that such an approach could improve the robustness of myoelectric control, and we provide preliminary results to confirm this assumption.

\section{MATERIALS AND METHODS}

\section{A. Novel classification approach}

Several studies of the human motor control point out to a consistency in the patterns of muscle activations characterizing different movements [3]. The same muscles are consistently recruited to perform a specific movement and with increasing intensity, their activations are scaled in the same relative proportions. If the muscle patterns are represented as vectors, where each coordinate represents the activation level of a single muscle, the patterns related to different movements are distributed in a specific manner in this vector space. They start from the origin (muscles relaxed) and then, with increasing contractions, the vectors radiate from the origin to populate a dedicated (hyper)conical area associated with each movement. This regularity in muscle activation patterns allows us to formulate a simple classification method. The intensity of muscle activation can be estimated using mean absolute value (MAV) of the segmented raw EMG. For each movement, a prototype vector is defined as the average of the vectors recorded during a single maximal voluntary contraction (MVC) lasting 3 seconds. For online control, the measured EMG vector is then classified into the movement class of the closest prototype vector, where the distance 
metric is the angle between the vectors. Hereafter, we name the novel method angular (ANG) classification.

\section{B. Experimental evaluation}

To evaluate the novel classification method, we have tested it in eleven able-bodied subjects (20-39 years) who operated a prosthesis to perform a set of functional tasks, including box and blocks, clothespin, bottle transfer and bottle turning task. We focus here on the bottle turning task, as this task is particularly challenging for the prosthesis use (grasping and manipulating a heavy object) and requires activating multiple movement classes (hand open and close, wrist rotation). The task for the subjects was to grasp a full 11 bottle placed vertically, rotate it and place it horizontally on the table. This was done for 3 bottles, one by one, and then the bottles were transferred from the horizontal back to the vertical position. The outcome measures were the time to successfully transfer three bottles back and forth (single round) and the number of bottles dropped during the transfer. The subjects performed four rounds in total (12 bottle transfers).

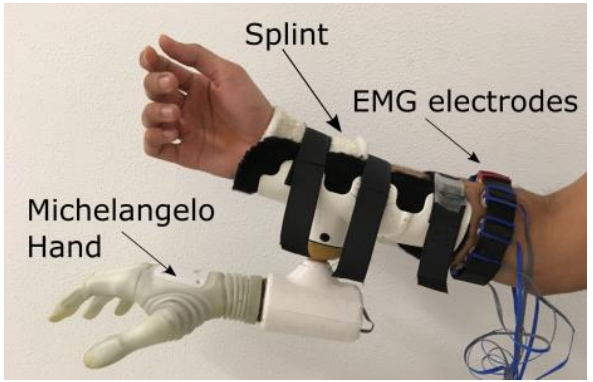

Fig. 1. The experimental setup: the Michelangelo hand with active wrist (pronation/supination) controlled via eight dry commercial EMG electrodes.

Eight commercial EMG electrodes (13E200 AC, Otto Bock) were embedded into a custom-made elastic brace placed around the forearm. The Michelangelo hand prosthesis (Otto Bock) with an active wrist was attached to a custom-made splint strapped around the forearm (Fig. 1). The classifier was trained to recognize four movements (hand opening and closing, wrist pronation and supination) and rest. The subjects performed the aforementioned tasks using the novel method and a popular benchmark, i.e., linear discriminant analysis (LDA) trained using a Hudgins timedomain feature set. The novel method was trained using MAV and MVC, as described previously, while the LDA was trained using three force levels for each movement (30, 60 and $90 \%$ of MVC), as recommended in the literature [4]. The order of the control methods was randomized across sessions. The results were compared using Wilcoxon signed rank test and the threshold for the statistical significance was set to $p<0.05$.

\section{RESULTS}

The summary results are given in Fig. 2. The results are reported in the text as median $(\mathrm{M})$ and interquartile range
(IQ), i.e., M/IQ. The subjects were significantly faster when performing the task using the novel method, i.e., the task completion time was 53/24 s for ANG versus 70/31 s per round for LDA. The control was also more robust as the subjects suffered significantly less drops per round.
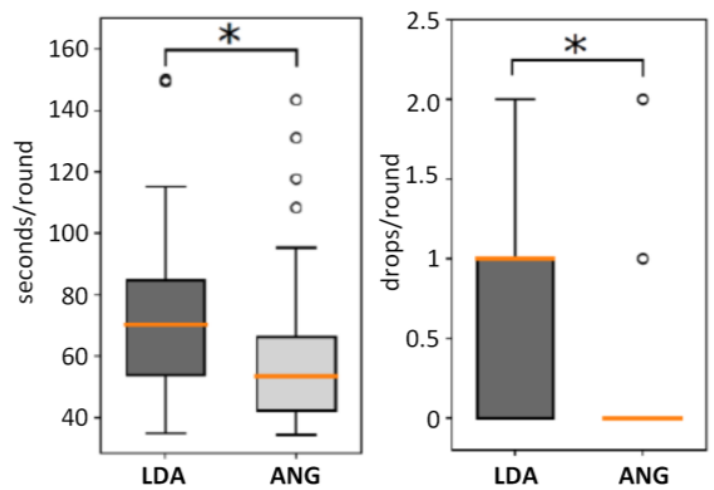

Fig. 2. Summary results for the block turn task. The box plots show the median (orange line), interquartile ranges (boxes), minimum and maximum (whiskers) and outliers (circle). The subjects performed the tasks faster and with less drops when using the novel method (ANG, light grey) compared to the benchmark (LDA, dark grey).

\section{DISCUSSION}

We have presented a novel method for classification of wrist and hand motions. The method exploits the natural coordination during activation of multiple muscles of the forearm. This coordination leads to a specific distribution of the amplitude features of the recorded EMG, and the proposed classification approach establishes the class boundaries using a specific metrics to capture this natural distribution. The preliminary results support the assumption that the novel method is more efficient and robust. When using the novel method, the subjects outperformed the widely used benchmark (LDA) in both outcome measures during a challenging functional task. Importantly, the improvement in performance was achieved despite the fact that the novel classification used less features and shorter training, i.e., MAV only versus Hudgins time-domain feature set, and MVC versus three force levels. Therefore, the new method also brings substantial benefits regarding shorter training and simple and efficient implementation.

\section{REFERENCES}

M. Asghari Oskoei and H. Hu, "Myoelectric control systems-A survey," Biomed. Signal Process. Control, vol. 2, no. 4, pp. 275294, Oct. 2007

[2] Ning Jiang, S. Dosen, K.-R. Muller, and D. Farina, "Myoelectric Control of Artificial Limbs: Is There a Need to Change Focus? [In the Spotlight]," IEEE Signal Process. Mag., vol. 29, no. 5, pp. 152-150, Sep. 2012.

[3] B. Poston, A. Danna-Dos Santos, M. Jesunathadas, T. M. Hamm and M. Santello, "Force-Independent Distribution of Correlated Neural Inputs to Hand Muscles During Three-Digit Grasping," J. Neurophysiol., vol. 104, no. 2, pp. 1141-1154, Aug. 2010.

[4] E. Scheme and K. Englehart, "Training Strategies for Mitigating the Effect of Proportional Control on Classification in Pattern Recognition-Based Myoelectric Control," JPO J. Prosthetics Orthot., vol. 25, no. 2, pp. 76-83, Apr. 2013. 